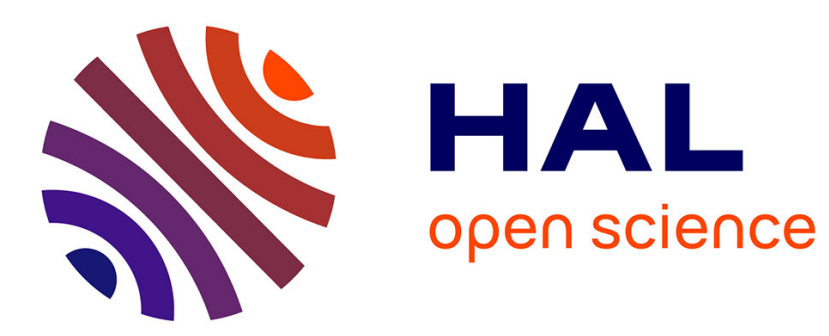

\title{
Response of Fish Gut Microbiota to Toxin-Containing Cyanobacterial Extracts: a Microcosm Study on the Medaka (Oryzias latipes)
}

Sébastien Duperron, Sebastien Halary, Myriam Habiballah, Alison Gallet, Hèlene H. Huet, Charlotte Duval, Cécile Bernard, Benjamin Marie

\section{To cite this version:}

Sébastien Duperron, Sebastien Halary, Myriam Habiballah, Alison Gallet, Hèlene H. Huet, et al.. Response of Fish Gut Microbiota to Toxin-Containing Cyanobacterial Extracts: a Microcosm Study on the Medaka (Oryzias latipes). Environmental Science and Technology, 2019, 10.1021/acs.estlett.9b00297 . mnhn-02434586

\section{HAL Id: mnhn-02434586}

\section{https://hal-mnhn.archives-ouvertes.fr/mnhn-02434586}

Submitted on 10 Jan 2020

HAL is a multi-disciplinary open access archive for the deposit and dissemination of scientific research documents, whether they are published or not. The documents may come from teaching and research institutions in France or abroad, or from public or private research centers.
L'archive ouverte pluridisciplinaire HAL, est destinée au dépôt et à la diffusion de documents scientifiques de niveau recherche, publiés ou non, émanant des établissements d'enseignement et de recherche français ou étrangers, des laboratoires publics ou privés. 
5 Sébastien Duperron ${ }^{1,2,+, *}$, Sébastien Halary ${ }^{1, \dagger}$, Myriam Habiballah ${ }^{1}$, Alison Gallet ${ }^{1}$, Hélène 6 Huet $^{3}$, Charlotte Duval ${ }^{1}$, Cécile Bernard ${ }^{1}$, Benjamin Marie ${ }^{1}$

$9 \quad{ }^{1}$ Muséum National d'Histoire Naturelle, CNRS, UMR7245 Mécanismes de Communication 10 et Adaptation des Micro-organismes, 12 rue Buffon, 75005 Paris, France.

$11{ }^{2}$ Institut Universitaire de France, 75005 Paris, France.

$12{ }^{3}$ École Nationale Vétérinaire d'Alfort, INRA - ANSES - ENVA UMR 1161 Virologie, 7 13 avenue du Général de Gaulle, 94704 Maisons-Alfort, France.

$15+$ the authors contributed equally to this work

$16{ }^{*}$ Corresponding author: sebastien.duperron@mnhn.fr 


\section{Abstract}

20 The effect of toxin-producing cyanobacterial blooms on fishes health has been investigated extensively, but no study to date has evaluated this effect on fish-associated microbiota. In this study, we test the effect of pure microcystins and of crude extracts of metabolites from Microcystis aeruginosa cultures on the composition of gut bacterial microbiota in Medaka

24 fishes (Oryzias latipes) exposed for 28 days in a microcosm experiment. A 16S rRNA-based 25 marker gene-based approach was used to investigate the composition of bacterial 26 communities. Results show that fish gut community compositions differ from those occurring 27 in the water, and among individual fishes. Exposure to extracts, rather than pure microcystin, 28 has a significant influence on gut community composition, with a marked increase in relative abundances of pathogen-related bacteria (genera Nocardia and Mycobacterium) in the presence of one extract, and of bacterial orders Sphingomonadales and Saprospirales in the other. We suggest that compounds identified in the cyanobacterial extracts, but not microcystin LR alone, alter the composition of bacterial communities, with possible consequences for various biological functions in fishes. This pioneer microcosm experiment indicates that cyanobacterial blooms probably have an effect on fish gut microbiota and associated functions, including toxin degradation and feed efficiency, and should be further explored. 
Cyanobacterial proliferations occur worldwide in freshwater, estuarine and marine environments, when environmental conditions allow (i.e. nutrient inputs, light intensity, high temperatures, $\mathrm{pH}$ and $\mathrm{CO}_{2}$ concentration). In recent years, massive cyanobacterial blooms increased in frequency and persistence around the world, causing serious threats to aquatic ecosystems ${ }^{1,2}$. Indeed, cyanobacteria produce numerous bio-active secondary metabolites, including various cyanotoxins, that remain and bioaccumulate into the environment, especially after bloom senescence ${ }^{3-5}$. The most widespread cyanotoxin is microcystin-leucinearginine (MC-LR) which can accumulate in the liver of aquatic vertebrates including fish ${ }^{6,7}$. Microcystins are produced, among others, by Microcystis, the most common cyanotoxinproducing and bloom-forming genus in freshwater ecosystems ${ }^{8}$.

Over the last decade, MC-LR effects upon fish ecotoxicology have been documented in some species $^{9-12 .}$. Among these, the Medaka fish (Oryzias latipes) is a model of choice for toxic effects investigation thanks to its resistance to stress and diseases. Natural cyanobacterial blooms, lab cultures, biomass extracts and pure microcystins (e.g. MC-LR) were shown to adversely impact its development, reproduction, and to induce specific organ alterations, notably hepatotoxicity and cardiotoxicity ${ }^{7,13-15}$. However, despite the documented impact of cyanobacterial blooms on fish health, no study has to our knowledge investigated the link between these events and the composition of fish microbiota.

In ecology, the holobiont, i.e. the super-organism composed by one pluricellular host and its associated microorganisms constituting its microbiota, is increasingly acknowledged as a relevant level of investigation ${ }^{16}$. Indeed, microbiota play multiple fundamental roles in host physiology, including in nutrition, immunity, protection and behavior ${ }^{17}$. In the context of aquaculture and fisheries, many investigations have recently highlighted the importance of fish microbiota for feed efficiency, and pathogen resistance ${ }^{18}$. Located at the interface between an organism and its environment, the microbiota is exposed to the latter, and should thus be accounted for in ecotoxicology studies. In this perspective, some cyanotoxins, known for their antimicrobial effect, could directly impact the composition of fish microbiota. Such effects have already been shown on the gut microbiota of the branchiopod Daphnia, for instance, resulting in alteration of their tolerance to toxic cyanobacteria ${ }^{19}$. Cyanobacterial secondary metabolites could also represent nutrient sources that select gut microbes able to use them. The aim of the present study is to test whether changes occur in fish microbiota compositions when hosts are exposed to cyanobacteria in a microcosm setup. As a natural cyanobacterial 
bloom is difficult to stimulate in the laboratory, we investigated the effects of three cyanobacterial extracts (two from distinct microcystin-producing Microcystis aeruginosa strains and one pure MC-LR extract) on water-borne bacterial populations and on the Medakas' gut microbiome composition. Cyanotoxins concentrations were chosen to mimic a low-level intoxication, just above the norm for drinking water ${ }^{20}$ to see how chronic exposure may impact gut bacteria. Bacterial communities are investigated using an Illumina-based metabarcoding approach on the V4-V5 region of the 16S rRNA-encoding gene. Potential

\section{Materials and methods}

\section{Secondary metabolites in extracts of Microcystis aeruginosa strains}

Three different metabolite mixtures were used in exposure experiments. The first consisted in pure microcystin-LR resuspended in 50\% ethanol/water solution and then partially evaporated (Novakit, France). The other two were extracted from cultures of Microcystis aeruginosa strains Paris Museum Collection (PMC) 728.11 (Extract 1) and Pasteur Culture Collection (PCC) 7820 (Extract 2) available upon request from the National Museum of Natural History and Institut Pasteur collections, respectively. Both were cultured at $25^{\circ} \mathrm{C}$ in $\mathrm{Z} 8$ media (Rippka 1988, 16h: $\left.8 \mathrm{~h} \mathrm{light/dark} \mathrm{cycle} \mathrm{at} 60 \mu \mathrm{mol} \cdot \mathrm{m}^{-2} \cdot \mathrm{s}^{-1}\right)$. Cell cultures were sonicated in methanol, centrifuged $\left(10 \mathrm{~min}, 4^{\circ} \mathrm{C}, 3,500 \mathrm{~g}\right)$, methanol was evaporated, extracts were resuspended (EtOH 50\%), and ethanol was evaporated. Microcystin concentration was measured as MCLR equivalents (MC-LR eq.) using the Novakit Microcystin-ADDA ELISA kit (France). Metabolites composition of the extracts were analyzed on an UHPLC (Ultimate 3000, ThermoFisher Scientific) coupled with a high-resolution mass spectrometer (ESI-Qq-TOF Maxis II ETD, Bruker). The analyte annotations were performed according to precise mass, isotopic and fragmentation MS/MS patterns, as previously described ${ }^{14}$.

\section{Exposure experiments and monitoring}

Adult male Medaka fishes were used in all experiments. Four treatments were applied: a solvent control without any cyanobacterial metabolite; exposure to Extract $1(\sim 2.5 \mu \mathrm{g}$ MC-LR eq. $\left.\mathrm{L}^{-1}\right)$; exposure to Extract $2\left(\sim 2.5 \mu \mathrm{g}\right.$ MC-LR eq. $\left.\mathrm{L}^{-1}\right)$; and exposure to pure microcystin $\left(\sim 2.5 \mu \mathrm{g}\right.$ MC-LR eq.L $\left.\mathrm{L}^{-1}\right)$. Each of the four treatments was applied on three replicate 10-liter aquaria, each containing six fishes that were acclimatized 10 days prior to the beginning of the exposure. Fishes were then exposed for 28 days at $25^{\circ} \mathrm{C} \pm 1{ }^{\circ} \mathrm{C}$. They were fed twice a day 
with T-0.3 Nutra HP (Skretting, Netherlands). Every two days, the aquaria were cleaned, half of the water was replaced, and the extracts or MC-LR were renewed in totality in the concentrations described above. Water temperature, conductivity, $\mathrm{pH}$, nitrate and nitrite were monitored every two days, and samples taken to further determine the total microcystin content using the ELISA kit.

\section{Fish dissection and tissue fixation}

At the end of the 28 days' exposure, three fishes $(n=3)$ were sampled from each of the 12 aquaria, except for aquaria $\mathrm{E}$ (four fishes), $\mathrm{F}$ and I (two fishes each, see Table S1). Fishes were anesthetized in $0.1 \%$ tricaine methane sulfonate (MS222; Sigma, St. Louis, MO), sacrificed, dissected, and the whole intestine (including content because of small size) was sampled and flash-frozen in liquid nitrogen. Aquarium water $(50 \mathrm{~mL})$ was filtered on a 0.22 $\mu \mathrm{m}$ nitrocellulose filter, then filters were flash frozen.

\section{DNA extraction and $16 S$ rRNA gene sequencing}

DNA was extracted from the intestine of 2 to 4 (mostly 3) replicate fishes from each aquarium, from water-filters and from fish food using a ZymoBIOMICS ${ }^{\mathrm{TM}}$ DNA mini kit (Zymo Research, CA), following mechanical lysis (bead-beater, 6 minutes, maximum speed). A $\sim 500$ bp fragment of the rRNA-encoding gene corresponding to the V4-V5 variable region of Escherichia coli was amplified using $515 \mathrm{~F}$ and $926 \mathrm{R}$ primers ${ }^{21}$ and sequenced on an Illumina MiSeq platform ( 2 X 250 bp, paired-end sequencing, Genoscreen, France). Raw reads were deposited into the GENBANK Sequence Read Archive (SRA) database under accession number PRJNA517613 (samples SAMN10839113 to SAMN10839169, see table $\mathrm{S} 2)$.

\section{Sequence analysis}

Raw reads were demultiplexed, quality checked and trimmed, and paired reads were assembled in contigs using $\mathrm{FLASH}^{22}$. Sequence analysis was performed using QIIME2 ${ }^{23}$. Amplicon Sequence Variants $\left(\mathrm{ASVs}^{24}\right.$ ) were identified using DEBLUR ${ }^{25}$. Chimeric sequences were identified using UCHIME (de novo chimera detection) and then removed ${ }^{26}$. Taxonomic affiliations were obtained by the sklearn-based classifier (GreenGenes 13-8-99 release). Sequences matching "Chloroplast" and "Mitochondria" were discarded. Rarefaction curves, alpha and beta diversity indices were generated using a sampling depth of 8,132 corresponding to the lowest number of quality-filtered reads obtained in a sample. Two gut 
samples (one from Extract 1 and one from Extract 2 experiments) were outliers in terms of (very low) ASVs number compared to samples from the same conditions, and were discarded from further analysis. A guide phylogenetic tree was produced to compute UniFrac distances ${ }^{27}$. Principal coordinates analysis (PCoA) plots based on Weighted and Unweighted UniFrac distances (WU and UU) as well as Bray Curtis (BC) dissimilarities were generated using ggplot $2^{28}$. Community richness were compared using ANOVA, and compositions were compared using PERMANOVA. A Venn diagram was drawn using the web-based software available at http://bioinformatics.psb.ugent.be/webtools/Venn/.

\section{Results and Discussion}

The analyzed parameters (temperature, conductivity, nitrites, $\mathrm{pH}$ and exposure levels measured as MC-LR equivalents) were stable within a given aquarium during the 28-days experiment (Table S1). Analysis of Extracts 1 and 2 from the M. aeruginosa strains revealed very different compositions in secondary metabolites besides MC-LR, present at various concentrations, including cyanopeptolins, aeruginosins, aerucyclamides, anabaenopeptins, microviridins and/or microginins (Figure S1). Exposure levels ranged between mean $1.1 \pm 0.4$ $\mu \mathrm{g}$ MC-LR eq.L ${ }^{-1}$ (Extract 1) and 5.6 $\pm 1.1 \mu \mathrm{g}$ MC-LR eq.L ${ }^{-1}$ (Extract 2), with $2.3 \pm 0.7 \mu \mathrm{g} . \mathrm{L}^{-1}$ in the pure MC-LR treatment. They were slightly above the norm for drinking water $(1.0 \mu \mathrm{g}$ eq. MC-LR eq. $\mathrm{L}^{-1}$ ), and represent chronic exposure levels rather than the acute levels expected during or after a very intense bloom event ${ }^{20}$. These conditions were previously shown to lead to observable lesions on the liver of young and adult fish, but not to major dysfunction ${ }^{14,29-31}$. The toxicity on hosts is thus limited, as are potential associated indirect effects on microbiota linked for example to organ malfunction leading to changes in the physico-chemical environment of microorganisms. Therefore, we expect to rather observe the direct effect of the MC-LR and the extracts on the microbiota itself.

A total of 1,861,481 assembled paired-end reads were obtained from 12 water samples (3 replicates per treatment) and 33 gut samples ( 7 to 9 per treatment, Table S1), of which $1,063,328$ from bacterial origin passed quality filters. Deconvolution resulted in a total of 662 ASVs. Rarefaction curves reached saturation for all samples, indicating that the dataset accurately represents the bacterial community (not shown). Depending on the sample, between 8,132 and 37,511 assembled sequences were obtained, representing 18 to 178 ASVs (Table S2). Comparisons indicate that the gut samples from specimens exposed to extracts 1 and 2 display overall higher diversity of ASVs and Shannon indices (Figure S2-3). 


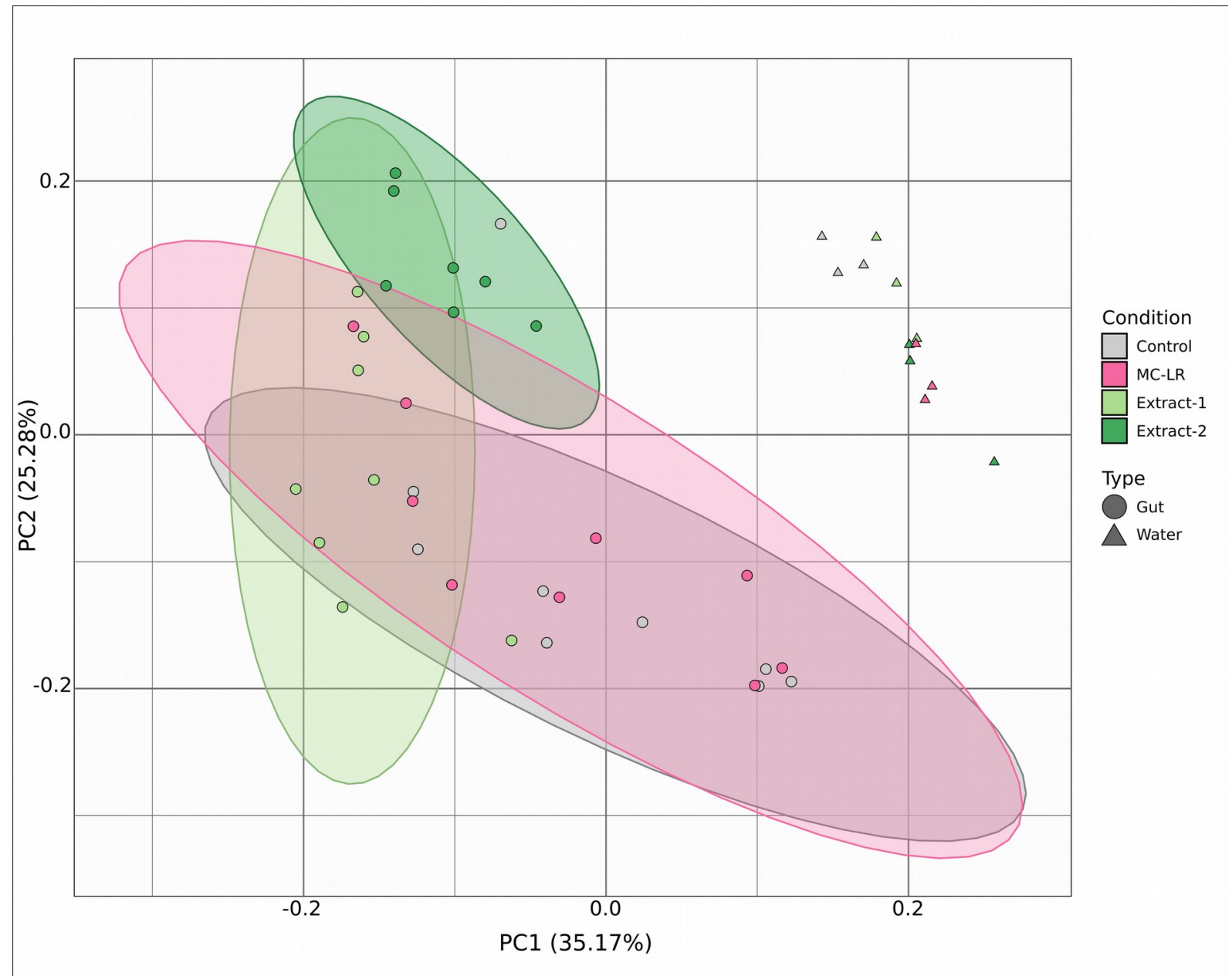

Figure 1. Principal Coordinates Analysis based on Weighted Unifrac dissimilarity metrics. Ellipses correspond to $95 \%$ confidence intervals for gut samples exposed to each of the four treatments.

\section{Bacterial communities in the water are similar}

Bacterial communities compositions were not significantly different among water samples whatever the treatment (WU distances, PERMANOVA $\mathrm{p}>0.09$ ), and clustered together in the principal coordinates analysis (PCoA, Figure 1). Similar results were observed in PcoA plots obtained using UU and BC dissimilarities (figure S4). These communities were dominated by Burkholderiales (Betaproteobacteria, mean $60.1 \pm 13.5 \%$ of reads), Saprospirales (Bacteroidetes, $11.8 \pm 6.3 \%$ ), Sphingomonodales $(8.1 \pm 4.6 \%)$ and Rickettsiales $(8.1 \pm 8.8 \%)$ (Alphaproteobacteria, Figure 2). Interestingly, abundances of Leptospirales (Spirochaetes), some of which are known to be animal pathogens, were significantly lower in the presence of cyanobacterial extracts or MC-LR compared to control ( $p$-value $<0.01$ ), suggesting selective antibacterial effects of these compounds. In total, the water samples displayed 390 ASVs, of which 145 also occurred in gut samples. 

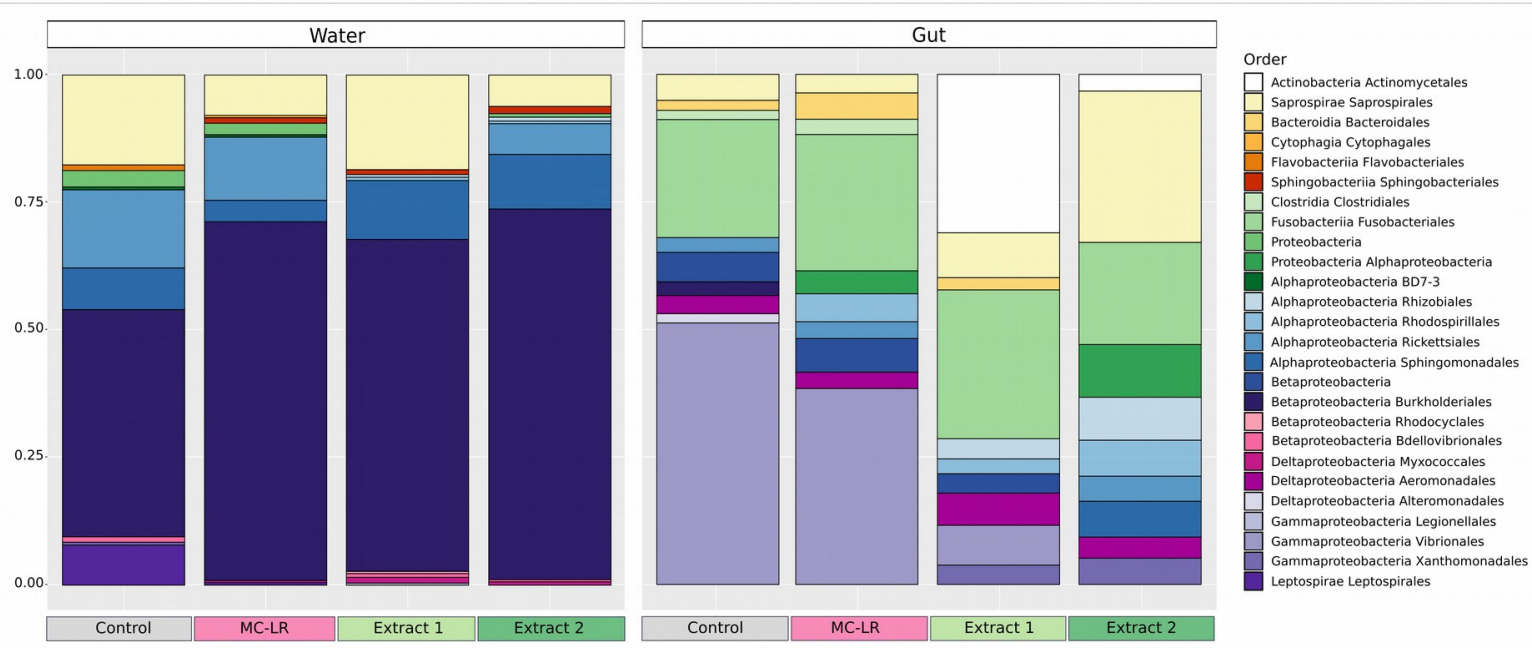

Figure 2. Relative abundances of major bacterial orders (mean values) present in water and gut samples in each treatment.

\section{Gut bacterial community composition is influenced by treatment}

Communities compositions from gut samples were different from those in the water (p-value $<0.001$ ) and well separated on the PCoA plots (Figure 1 and S4). Differences between fish gut and water communities have previously been reported in various studies ${ }^{18,32-34}$. In contrast to water, gut bacterial communities were much more spread on the PCoA plot (Figure 1), emphasizing high inter-individual variability within a given experimental condition. This variability is commonly observed among vertebrate hosts ${ }^{34-37}$ and is not explained by the different aquaria used in this study (factor "aquarium" on gut community composition, pvalue $>0.4$ ). Bacterial community compositions were not significantly different between control and MC-LR-exposed fish guts ( $>0.7$, Figure 1). These on the other hand strongly differed from compositions in guts of fish exposed to Extract 1 and Extract 2 (p-values $<0.01$ ), which also differed between them $(p$-value $<0.01)$. The same results were obtained in UU and $\mathrm{BC}$ analyses (figure S4). 


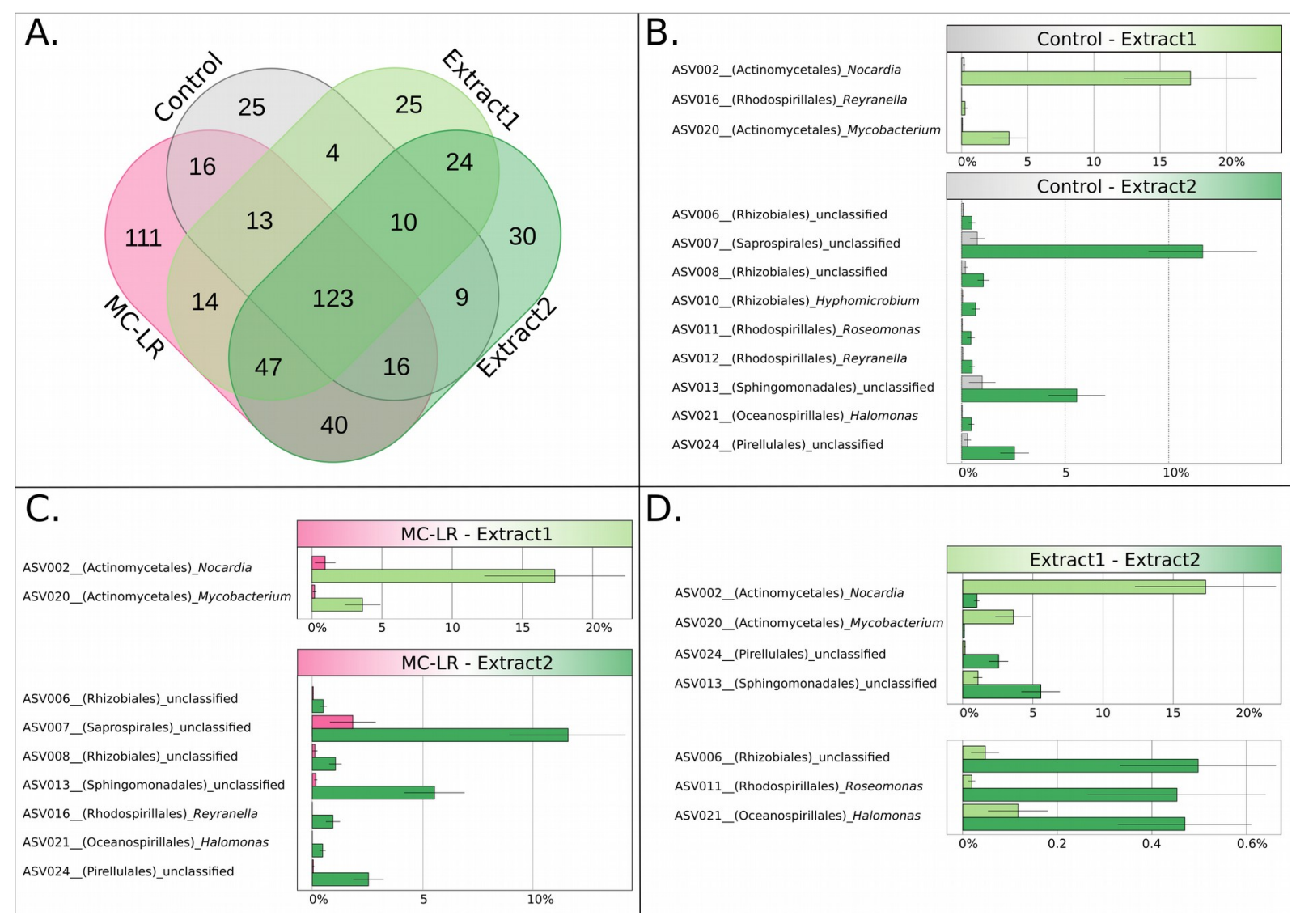

Figure 3. A. Venn Diagram displaying the number of gut bacterial ASVs shared among treatments. B-D: Major gut ASVs displaying significant differences in relative abundance between two conditions (pairwise t-tests with Bonferroni correction, $\mathrm{p}$-values $<0.01$ ). The bar plots display the mean proportion of ASV in gut samples from each of the two treatments, named by their ID and their affiliated genus, when available. Among gut ASVs, 26 displayed significantly different relative abundances between at least two treatments, of which only the 12 that represented at least $1 \%$ of reads in at least one gut sample are listed.

\section{Identity of stable and variable taxa and possible link with treatments}

Overall, 507 distinct ASVs occurred in gut samples, of which 123 were shared among all treatments and 25 to 111 were unique to one treatment (Figure 3A). The 20 most abundant ASVs represented between 53.7 and $97.8 \%$ of total reads in a given individual. In each bacterial order, a single or two ASVs represented most of the reads. Significant variations in taxa relative abundances were observed among treatments (summarized at the Class, Order, Family and Genus levels in figure S5, and for ASVs in figure 3). Despite variation among treatments, order Fusobacteriales (Fusobacteria), dominated by a single ASV affiliated to Cetobacterium somerae (ASV253), was abundant in fish gut microbiota in all conditions (mean reads per treatment between $12.9 \%$ and $26.2 \%$, Figure 2). C. somerae notably produces B12 vitamins beneficial to various hosts and plays an essential role in healthy host physiology in several species ${ }^{32,38-40}$. Vibrionales (Gammaproteobacteria) represented by two main ASVs, 
namely ASV220 and ASV378, was the second most abundant bacterial order in three treatments (mean reads per treatment between $7.9 \%$ and $46.3 \%$ ), but was almost absent in guts from fishes exposed to Extract 2 (mean=0.17\%, Figure 2, Table S3, Figure S5). This order includes commensal and animal pathogenic genera which are common in fish guts $36,39,40$. Both taxa, C. somerae and Vibrionales, have already been reported in congeneric Oryzias melastigma (marine Medaka), and in a small 16S rRNA clone library obtained from O. latipes $\operatorname{gut}^{39,40}$.

Various other taxa were related to common fish pathogens, for example Aeromonas salmonicida (ASV237, mean $2.9 \%$ of reads over all treatments ${ }^{34}$ ). Among these, two ASVs within Class Actinobacteria were significantly more abundant in guts exposed to Microcystis aeruginosa Extract 1 compared to Extract 2, Control and MC-LR treatments (Figure 3B-D, Figure S5). The first ASV was related to genus Nocardia, namely ASV002 (mean $17.2 \%$ of reads in Extract 1 samples, occuring in 7 of the 8 specimens), and the second to genus Mycobacterium (ASV020, 3.6\% of reads in Extract 1 samples, present in 7 out of 8 specimens). Nocardia and Mycobacterium genera, both belonging to the family Mycobacteriaceae, are known as pathogens of animals, including fish ${ }^{41}$. Interestingly, Mycobacteria are frequently reported to be more abundant in guts of animals suffering inflammatory bowel diseases even though their role in these pathologies remains unclear ${ }^{42,43}$. Nevertheless, dysbioses (i.e. lasting changes of the microbiota), as those occuring in inflammatory bowel diseases, are known to favor the increase of bacterial pathogens abundances by disrupting the protective barrier provided by the healthy gut microbiota ${ }^{44,45}$. Therefore, the change in bacterial community and the increase of Mycobacteria abundances may reflect a dysbosis induced by chronic exposure to Extract 1 (Figure S5).

In Extract 2 treatment, two other bacterial orders displayed significantly higher abundances compared to other treatments, namely Saprospirales (ASV007, 11.6\% of reads in Extract 2 samples) and Sphingomonadales (ASV013, 5.5\% of reads, Figures 2, 3B and 3C), and ASVs were present in all specimens. These orders are not reported to include animal pathogens, but some are able to degrade a wide variety of metabolites including complex carbohydrates, proteins and aromatic compounds ${ }^{46}$. For instance, plant-based diets promote Saprospirales population in fish guts ${ }^{47}$. Growth of Sphingomonadales members has been shown to correlate with that of cyanobacteria including Microcystis, and the former were suspected to benefit from organic matter produced by cyanobacteria ${ }^{48,49}$. Furthermore, several members of Sphingomonadales can use microcystins as nutrient sources ${ }^{48}$. Overall, observed increase of Saprospirales and Sphingomonadales abundances thus suggests that Extract 2 
exposure selected microbiota members able to metabolize cyanobacterial secondary metabolites. By modulating gut microbiota compositions, relatively low cyanobacterial metabolites abundances could thus have serious effects on host feed efficiency.

Overall, results from this study first show that MC-LR alone had no noticeable effect on the composition of gut-associated bacterial communities as recently shown on pooled individuals of zebrafish ${ }^{50}$, at the levels and duration applied here, while full extracts of Microcystis aeruginosa both had a significant effect. Full extracts contained various secondary metabolites besides MCs, among which some probably directly affect animalassociated microbial communities, either alone or together. For instance, aerucyclamides, some of which were identified here (Figure S1), are cyclic peptides that may present antimicrobial and cytotoxic activities. Cyanobacterial secondary metabolites may also include compounds interfering with bacterial quorum sensing, or exhibiting allelopathic or antibacterial properties ${ }^{51}$. These compounds may lead to dysbioses and their consequences, such as the increase in abundances of potential pathogens observed in Extract 1, or the increase in bacterial taxa that may use cyanobacterial metabolites as hypothesized in Extract 2. Possible functional consequences for fish health need to be further explored, but these changes in microbiota composition could alter feed efficiency, pathogen susceptibility or protective functions of the gut. Experiments from this study mimic what may happen in a lake after the end of a bloom, when compounds are released in the water following cyanobacteria senescence. Given the reported consequences of blooms for fish health in the wild and in aquaculture settings where they may greatly affect yields, this topic appears as a promising line of research in animal-bacteria interactions and a microcosm-based approach using extracts is an appropriate first step to tackle the issue. The next step will be to investigate the effect of direct exposure to cyanobacterial blooms, ideally in mesocosm setups, to test whether the actual presence of bacteria modifies these effects.

\section{Funding}

The study was funded by MNHN through a grant to MH (UMR 7245) and ATM grant 3M awarded to $\mathrm{SD}$ and $\mathrm{BM}$. AG is funded through a $\mathrm{PhD}$ grant from Ecole Doctorale 227 "Sciences de la Nature et de l'Homme", MNHN.

\section{Supporting Information Available}


Supporting information contains additional data and analyses on the composition of cyanobacterial extracts, alpha diversity (observed ASVs and Shannon indices), beta diversity (PcoA plots and tests based on UU and BC metrics), and community composition at the Class, parameters, samples ID and characteristics, and relative abundances, taxonomic affiliation and sequences of all ASVs. This information is available free of charge on the ACS Publications

\section{Authors contribution}

$\mathrm{SD}$ and $\mathrm{BM}$ conceived the study

$\mathrm{MH}, \mathrm{CD}, \mathrm{BM}$ and SD conducted the exposure experiments

$\mathrm{SD}, \mathrm{SH}, \mathrm{AG}, \mathrm{BM}$ and $\mathrm{MH}$ performed molecular analyses, and analyzed the data

$\mathrm{HH}$ performed the analyses on liver.

\section{Ethics statement}

Experiments on fishes were conducted according to good practice, validated by comité Cuvier (Author. APAFiS\#19316-2019032913284201) and under the supervision of accredited personnel (CD, BM).

\section{Acknowledgments}

We thank Dr. A. Escalas for his help with statistical analyses of the data.

\section{References}

(1) Huisman, J.; Codd, G. A.; Paerl, H. W.; Ibelings, B. W.; Verspagen, J. M. H.; Visser, P. M. Cyanobacterial Blooms. Nat. Rev. Microbiol. 2018, 16, 471-483.

(2) Paerl, H. W.; Otten, T. G. Harmful Cyanobacterial Blooms: Causes, Consequences, and Controls. Microb. Ecol. 2013, 65, 995-1010.

(3) Leflaive, J.; Ten-Hage, L. Algal and Cyanobacterial Secondary Metabolites in Freshwaters: A Comparison of Allelopathic Compounds and Toxins. Freshw. Biol. 2007, 52, 199-214.

(4) van Apeldoorn, M. E.; van Egmond, H. P.; Speijers, G. J. A.; Bakker, G. J. I. Toxins of Cyanobacteria. Mol. Nutr. Food Res. 2007, 51, 7-60.

(5) Flores, N. M.; Miller, T. R.; Stockwell, J. D. A Global Analysis of the Relationship between Concentrations of Microcystins in Water and Fish. Front. Mar. Sci. 2018, 5, 30.

(6) Malbrouck, C.; Kestemont, P. Effects of Microcystins on Fish. Environ. Toxicol. Chem. 2006, 25, 72-86.

(7) Qiao, Q.; Le Manach, S.; Huet, H.; Duvernois-Berthet, E.; Chaouch, S.; Duval, C.; Sotton, B.; Ponger, L.; Marie, A.; Mathéron, L.; et al. An Integrated Omic Analysis of Hepatic Alteration in Medaka Fish Chronically Exposed to Cyanotoxins with Possible Mechanisms of Reproductive 
Toxicity. Environ. Pollut. 2016, 219, 119-131.

(8) Harke, M. J.; Steffen, M. M.; Gobler, C. J.; Otten, T. G.; Wilhelm, S. W.; Wood, S. A.; Paerl, H. W. A Review of the Global Ecology, Genomics, and Biogeography of the Toxic Cyanobacterium, Microcystis Spp. Harmful Algae 2016, 54, 4-20.

(9) Wang, P.-J.; Chien, M.-S.; Wu, F.-J.; Chou, H.-N.; Lee, S.-J. Inhibition of Embryonic Development by Microcystin-LR in Zebrafish, Danio Rerio. Toxicon Off. J. Int. Soc. Toxinology 2005, 45 , 303-308.

(10) Xie, L.; Xie, P.; Guo, L.; Li, L.; Miyabara, Y.; Park, H.-D. Organ Distribution and Bioaccumulation of Microcystins in Freshwater Fish at Different Trophic Levels from the Eutrophic Lake Chaohu, China. Environ. Toxicol. 2005, 20, 293-300.

(11) Zhang, D.; Xie, P.; Liu, Y.; Qiu, T. Transfer, Distribution and Bioaccumulation of Microcystins in the Aquatic Food Web in Lake Taihu, China, with Potential Risks to Human Health. Sci. Total Environ. 2009, 407, 2191-2199.

(12) Pavagadhi, S.; Balasubramanian, R. Toxicological Evaluation of Microcystins in Aquatic Fish Species: Current Knowledge and Future Directions. Aquat. Toxicol. Amst. Neth. 2013, 142143, 1-16.

(13) Trinchet, I.; Djediat, C.; Huet, H.; Dao, S. P.; Edery, M. Pathological Modifications Following Sub-Chronic Exposure of Medaka Fish (Oryzias latipes) to Microcystin-LR. Reprod. Toxicol. Elmsford N 2011, 32, 329-340.

(14) Le Manach, S.; Sotton, B.; Huet, H.; Duval, C.; Paris, A.; Marie, A.; Yépremian, C.; Catherine, A.; Mathéron, L.; Vinh, J.; et al. Physiological Effects Caused by Microcystin-Producing and NonMicrocystin Producing Microcystis Aeruginosa on Medaka Fish: A Proteomic and Metabolomic Study on Liver. Environ. Pollut. Barking Essex 1987 2018, 234, 523-537.

(15) Saraf, S. R.; Frenkel, A.; Harke, M. J.; Jankowiak, J. G.; Gobler, C. J.; McElroy, A. E. Effects of Microcystis on Development of Early Life Stage Japanese Medaka (Oryzias Latipes): Comparative Toxicity of Natural Blooms, Cultured Microcystis and Microcystin-LR. Aquat. Toxicol. Amst. Neth. 2018, 194, 18-26.

(16) Zilber-Rosenberg, I.; Rosenberg, E. Role of Microorganisms in the Evolution of Animals and Plants: The Hologenome Theory of Evolution. FEMS Microbiol. Rev. 2008, 32, 723-735.

(17) McFall-Ngai, M.; Hadfield, M. G.; Bosch, T. C. G.; Carey, H. V.; Domazet-Loso, T.; Douglas, A. E.; Dubilier, N.; Eberl, G.; Fukami, T.; Gilbert, S. F.; et al. Animals in a Bacterial World, a New Imperative for the Life Sciences. Proc. Natl. Acad. Sci. U. S. A. 2013, 110, 3229-3236.

(18) Llewellyn, M. S.; Boutin, S.; Hoseinifar, S. H.; Derome, N. Teleost Microbiomes: The State of the Art in Their Characterization, Manipulation and Importance in Aquaculture and Fisheries. Front. Microbiol. 2014, 5, 207.

(19) Macke, E.; Callens, M.; Meester, L. D.; Decaestecker, E. Host-Genotype Dependent Gut Microbiota Drives Zooplankton Tolerance to Toxic Cyanobacteria. Nat. Commun. 2017, 8, 1608.

(20) Chorus, I.; Bartram, J. Toxic Cyanobacteria in Water : A Guide to Their Public Health Consequences, Monitoring and Management; Geneva : World Health Organization: Geneva, 1999.

(21) Parada, A. E.; Needham, D. M.; Fuhrman, J. A. Every Base Matters: Assessing Small Subunit rRNA Primers for Marine Microbiomes with Mock Communities, Time Series and Global Field Samples. Environ. Microbiol. 2016, 18, 1403-1414.

(22) Magoč, T.; Salzberg, S. L. FLASH: Fast Length Adjustment of Short Reads to Improve Genome Assemblies. Bioinforma. Oxf. Engl. 2011, 27, 2957-2963.

(23) Hall, M.; Beiko, R. G. 16S RRNA Gene Analysis with QIIME2. Methods Mol. Biol. Clifton NJ 2018, 1849, 113-129.

(24) Callahan, B.J; McMurdie, P.J.; Holmes, S.P. Exact sequence variants should replace operational taxonomic units in marker-gene data analysis. ISME J. 2017, 11, 2638-2643.

(25) Amir, A.; McDonald, D.; Navas-Molina, J. A.; Kopylova, E.; Morton, J. T.; Zech Xu, Z.; Kightley, E. P.; Thompson, L. R.; Hyde, E. R.; Gonzalez, A.; et al. Deblur Rapidly Resolves Single-Nucleotide Community Sequence Patterns. MSystems 2017, 2, e00191-16. .

(26) Edgar, R. C.; Haas, B. J.; Clemente, J. C.; Quince, C.; Knight, R. UCHIME Improves Sensitivity and 
Speed of Chimera Detection. Bioinforma. Oxf. Engl. 2011, 27, 2194-2200.

(27) Lozupone, C.; Knight, R. UniFrac: A New Phylogenetic Method for Comparing Microbial Communities. Appl. Environ. Microbiol. 2005, 71, 8228-8235.

(28) Oksanen, J.; Kindt, R.; Legendre, P.; O'Hara, B.; Stevens, M. H. H.; Oksanen, M. J.; Suggests, M. The Vegan Package. Community Ecol. Package 2007, 1, 631-637.

(29) Chen, L.; Hu, Y.; He, J.; Chen, J.; Giesy, J.P.; Xie, P. Responses of the proteome and metabolome in livers of zebrafish exposed chronically to environmentally relevant concentrations of microcystin-LR. Environ. Sci. Technol. 2017, 51, $596 \mathrm{e} 607$.

(30) Hou, J.; Li, L.; Wu, N.; Su, Y.; Lin,W.; Li, G.; Gu, Z. Reproduction impairment and endocrine disruption in female zebrafish after long-term exposure to MC-LR: a life cycle assessment. Environ. Pollut. 2016, 208, 477e485.

(31) Le Manach, S.; Khenfech, N.; Huet, H.; Qiao, Q.; Duval, C.; Marie, A.; Bolbach, G.; Clodic, G.; Djediat, C.; Bernard, C.; Edery, M.; Marie, B. Gender-specific toxicological effects of chronic exposure to pure microcystin-LR or complex Microcystis aeruginosa extracts on adult medaka fish. Environ. Sci. Technol. 2016, 50, 8324e8334.

(32) Liu, H.; Guo, X.; Gooneratne, R.; Lai, R.; Zeng, C.; Zhan, F.; Wang, W. The Gut Microbiome and Degradation Enzyme Activity of Wild Freshwater Fishes Influenced by Their Trophic Levels. Sci. Rep. 2016, 6, 24340.

(33) Yan, Q.; Li, J.; Yu, Y.; Wang, J.; He, Z.; Nostrand, J. D. V.; Kempher, M. L.; Wu, L.; Wang, Y.; Liao, L.; et al. Environmental Filtering Decreases with Fish Development for the Assembly of Gut Microbiota. Environ. Microbiol. 2016, 18, 4739-4754.

(34) de Bruijn, I.; Liu, Y.; Wiegertjes, G. F.; Raaijmakers, J. M. Exploring Fish Microbial Communities to Mitigate Emerging Diseases in Aquaculture. FEMS Microbiol. Ecol. 2018, 94, fix161.

(35) Lozupone, C. A.; Stombaugh, J. I.; Gordon, J. I.; Jansson, J. K.; Knight, R. Diversity, Stability and Resilience of the Human Gut Microbiota. Nature 2012, 489, 220-230.

(36) Star, B.; Haverkamp, T. H. A.; Jentoft, S.; Jakobsen, K. S. Next Generation Sequencing Shows High Variation of the Intestinal Microbial Species Composition in Atlantic Cod Caught at a Single Location. BMC Microbiol. 2013, 13, 248.

(37) Stephens, W. Z.; Burns, A. R.; Stagaman, K.; Wong, S.; Rawls, J. F.; Guillemin, K.; Bohannan, B. J. M. The Composition of the Zebrafish Intestinal Microbial Community Varies across Development. Isme J. 2016, 10, 644-654.

(38) Tsuchiya, C.; Sakata, T.; Sugita, H. Novel Ecological Niche of Cetobacterium somerae, an Anaerobic Bacterium in the Intestinal Tracts of Freshwater Fish. Lett. Appl. Microbiol. 2008, 46, 4348.

(39) Givens, C. E.; Ransom, B.; Bano, N.; Hollibaugh, J. T. Comparison of the Gut Microbiomes of 12 Bony Fish and 3 Shark Species. Mar. Ecol. Prog. Ser. 2015, 518, 209-223.

(40) Chen, L.; Lam, J. C. W.; Hu, C.; Tsui, M. M. P.; Wang, Q.; Giesy, J. P.; Lam, P. K. S. Perfluorobutanesulfonate Exposure Causes Durable and Transgenerational Dysbiosis of Gut Microbiota in Marine Medaka. Environ. Sci. Technol. Lett. 2018, 5, 731-738.

(41) Kim, B. S.; Park, J. W.; Kang, G. S.; Jin, J. H.; Roh, H. J.; Kim, D. H.; Lee, M. K.; Huh, M. D. First Report of Nocardia Infection in Cultured Japanese Eel, Anguilla japonica. J. Fish Dis. 2018, 41, 1921-1927.

(42) Subramanian, S.; Campbell, B. J.; Rhodes, J. M. Bacteria in the Pathogenesis of Inflammatory Bowel Disease. Curr. Opin. Infect. Dis. 2006, 19, 475-484.

(43) Fecteau, M.-E. Paratuberculosis in Cattle. Vet. Clin. North Am. Food Anim. Pract. 2018, 34, 209-222.

(44) Hoarau, G.; Mukherjee, P. K.; Gower-Rousseau, C.; Hager, C.; Chandra, J.; Retuerto, M. A.; Neut, C.; Vermeire, S.; Clemente, J.; Colombel, J. F.; et al. Bacteriome and Mycobiome Interactions Underscore Microbial Dysbiosis in Familial Crohn's Disease. MBio 2016, 7, e01250-16.

(45) Selber-Hnatiw, S.; Rukundo, B.; Ahmadi, M.; Akoubi, H.; Al-Bizri, H.; Aliu, A. F.; Ambeaghen, T. U.; Avetisyan, L.; Bahar, I.; Baird, A.; et al. Human Gut Microbiota: Toward an Ecology of Disease. Front. Microbiol. 2017, 8, 1265. 
(46) Thomas, F.; Hehemann, J.-H.; Rebuffet, E.; Czjzek, M.; Michel, G. Environmental and Gut Bacteroidetes: The Food Connection. Front. Microbiol. 2011, 2, 93.

(47) Michl, S. C.; Beyer, M.; Ratten, J.-M.; Hasler, M.; LaRoche, J.; Schulz, C. A Diet-Change Modulates the Previously Established Bacterial Gut Community in Juvenile Brown Trout (Salmo trutta). Sci. Rep. 2019, 9, 2339.

(48) Ishii, H.; Nishijima, M.; Abe, T. Characterization of Degradation Process of Cyanobacterial Hepatotoxins by a Gram-Negative Aerobic Bacterium. Water Res. 2004, 38, 2667-2676.

(49) Parveen, B.; Ravet, V.; Djediat, C.; Mary, I.; Quiblier, C.; Debroas, D.; Humbert, J.-F. Bacterial Communities Associated with Microcystis Colonies Differ from Free-Living Communities Living in the Same Ecosystem. Environ. Microbiol. Rep. 2013, 5, 716-724.

(50) Li, J.; Chen, C.; Zhang, T.; Liu, W.; Wang, L.; Chen, Y.; Wu, L.; Hegazy, A.M.; El-Sayed, A.F.; Zhang, X. $\mu$ Evaluation of microcysti-LR absorbtion using and in vivo intestine model and its effect on zebrafish intestine. Aquat. Toxicol. 2019, 206, 186-194.

(51) Shah, S. A. A.; Akhter, N.; Auckloo, B. N.; Khan, I.; Lu, Y.; Wang, K.; Wu, B.; Guo, Y.-W. Structural Diversity, Biological Properties and Applications of Natural Products from Cyanobacteria. A Review. Mar. Drugs 2017, 15, E354. 


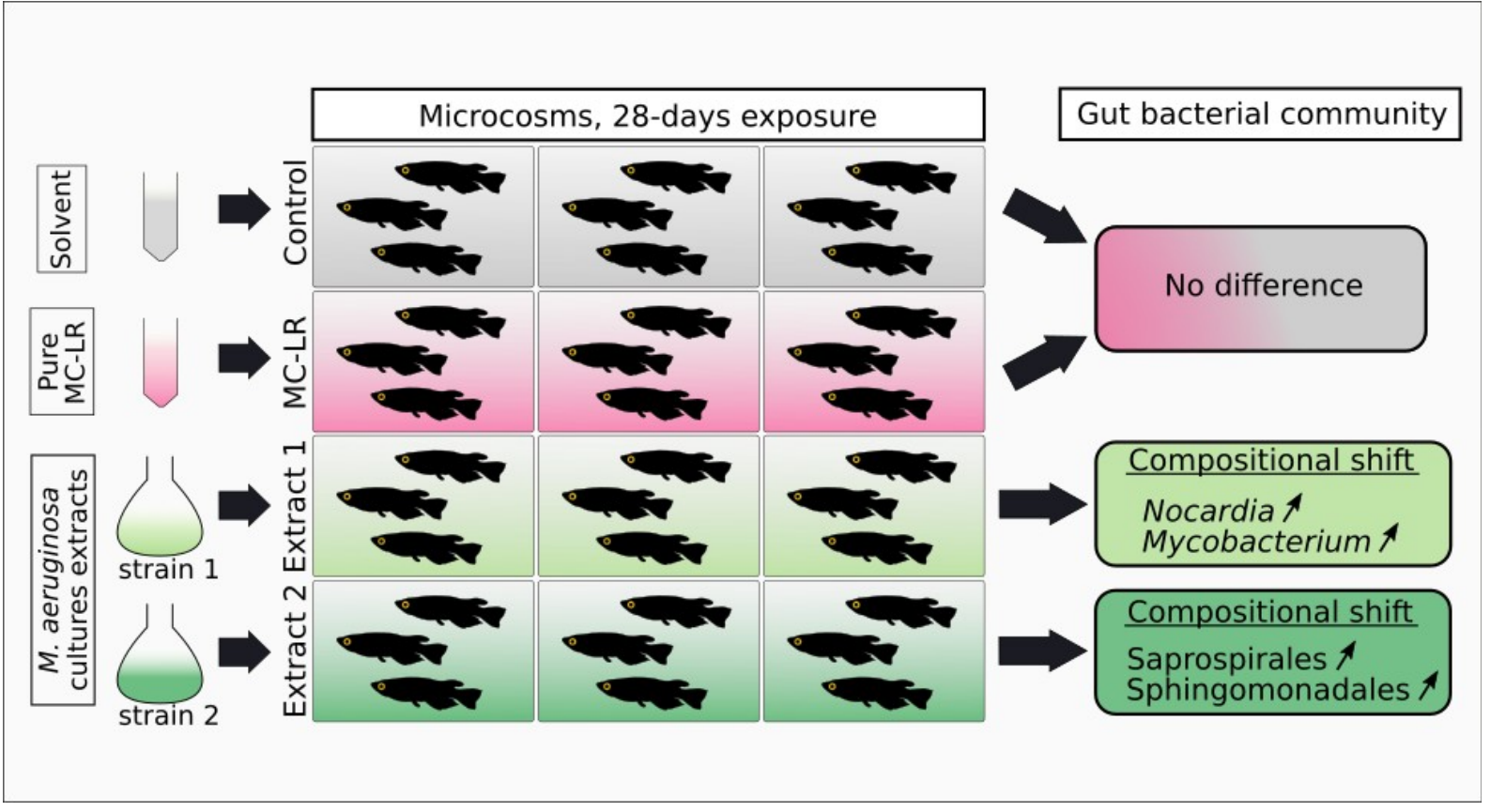

